joints were collected for inflammatory cytokine (IL-1ß and KC) determination and histological analysis, respectively.

Results: The mean change in ankle swelling after i.a injection was $0.595 \pm 0.434 \mathrm{~mm}$. Prophylactic treatment with PD and colchicine significantly diminished ankle swelling to $0.175 \pm 0.115 \mathrm{~mm}$ and $0.137 \pm 0.100 \mathrm{~mm}$, respectively (Kruskal Wallis $p$ 0.0025; Dunn's post test $p<0.01$ CPP vs PD+CPP). The therapeutic administration of PD did not have significant effects on delta swelling $(0.468 \pm 0.372 \mathrm{~mm}$ - PD vs $0.243 \pm 0.152 \mathrm{~mm}$ - colchicine). In mice treated with CPP crystals, histological analysis revealed areas of edema and increased cell infiltrate in articular and periarticular tissues and the presence of reactive lymphnodes. Tissue necrosis around inflamed tissue has been observed. Treatment with PD importantly reduced cell infiltrate in the prophylactic but not in the therapeutic protocol.

Serum IL-1ß and KC levels, which increased significantly $(p<0.05)$ after $48 \mathrm{~h}$ from i.a injection, diminished in non significant manner after prophylactic and therapeutic treatment. The gene expression study revealed a reduction of IL-1B and KC mRNA after PD and colchicine treatment in both groups.

Conclusion: PD can effectively prevent acute inflammatory response to crystals in the mouse model of CPP arthritis. Oral PD prophylactic treatment showed a similar effect of colchicine in reducing ankle swelling and cell infiltrate. However, only colchicine showed to be effective in the therapeutic protocol.

These results raise the possibility that PD might have utility in the prevention of crystal-induced acute attacks in humans.

References:

[1] Oliviero $\mathrm{F}$, et al. Polydatin and resveratrol inhibit the inflammatory process induced by urate and pyrophosphate crystals in thp-1 cells. Foods $2019 \mathrm{Nov}$ 7;8(11). pii: E560.

Disclosure of Interests: Francesca Oliviero: None declared, Francesca Galuppini: None declared, Anna Scanu: None declared, Paola Galozzi: None declared, Vanni Lazzarin: None declared, Paolo Sfriso: None declared, Gianpietro Ravagnan: None declared, Roberta Ramonda Speakers bureau: Novartis, Celgene, Janssen, Pfizer, Abbvie, Lilly, Paolo Spinella: None declared, LEONARDO PUNZI: None declared, Gianmaria Pennelli: None declared, Roberto Luisetto: None declared

DOI: 10.1136/annrheumdis-2020-eular.2124

\section{OP0175 \\ IDENTIFYING PERIPHERAL VASCULAR MONOSODIUM URATE CRYSTAL DEPOSITION WITH DUAL-ENERGY CT: FACT OR FICTION? THE VASCURATE STUDY}

T. Pascart ${ }^{1}$, P. Carpentier ${ }^{1}$, L. Norberciak ${ }^{1}$, J. Legrand ${ }^{1}$, E. Houvenagel ${ }^{1}$, F. Becce ${ }^{2}$, J. F. Budzik ${ }^{1}{ }^{1}$ Groupe Hospitalier de L'institut Catholique de Lille, Lille, France; ${ }^{2} \mathrm{CHUV}$ Lausanne, Lausanne, France

Background: The close relationship between gout and cardiovascular diseases is well established. A growing hypothesis explaining this association would be that monosodium urate (MSU) crystals are deposited within vessel walls. Dual-energy computed tomography (DECT) can identify and quantify MSU crystal deposition in soft tissues. It remains unclear whether vascular spots exhibiting DECT attenuation characteristics of MSU are artefacts or true MSU crystal deposits.

Objectives: The objectives of this study were to determine whether the presence of peripheral vascular MSU crystal deposition identified with DECT is associated with the extent of MSU deposits in joint soft tissues, and if this association persists over time under urate-lowering therapy.

Methods: Patients with a clinical suspicion or established gout diagnosis prospectively underwent DECT for identification and quantification of the MSU crystal burden in their knees and feet. Some of these patients were also enrolled in the GOUT-DECTUS longitudinal study, and thus underwent follow-up DECT scans of their knees and feet at 6, 12 and 24 months. DECT scans were examined for the presence of vascular spots $\geq 0.01 \mathrm{~cm} 3$ classified as MSU crystal deposits according to the default post-processing settings. Multiple linear regressions adjusting on serum urate levels and gout diagnosis were implemented to determine the association between DECT MSU crystal volume in joint soft tissues, and the presence of vascular MSU deposits. Mixed linear models were used to compare DECT volumes of MSU crystal deposition in soft tissues between vascular MSU positive and negative patients during follow-up.

Results: A total of 169 patients were included, of which 140 had a final diagnosis of gout, including 15 also included in the longitudinal study. Patients were mostly male $(78.8 \%)$ and were $65.5 \pm 14.6$ years old. Among gout patients, disease duration was $9.3 \pm 9.9$ years and $56.5 \%$ were urate lowering therapy-naive. A total of $11 / 29(37.9 \%)$ controls and $40 / 140(28.6 \%)$ gout patients presented with a least one vascular spot of DECT MSU deposition, with an average volume of $0.02 \pm 0.02 \mathrm{~cm} 3$, and all subjects also presented at least one vascular calcification. In the feet, patients positive for vascular DECT MSU crystal deposition had an MSU volume of $3.81 \pm 10.06 \mathrm{~cm} 3$ in joint soft tissues, compared with
$1.85 \pm 7.72 \mathrm{~cm} 3$ for those without vascular MSU deposition $(p=0.018)$. In the knees, patients with vascular MSU deposition had an MSU crystal volume of $6.03 \pm 24.13 \mathrm{~cm} 3$ in joint soft tissues, compared with $0.83 \pm 2.88 \mathrm{~cm} 3$ for those without vascular evidence of MSU deposition. In the longitudinal subgroup analysis, coefficients of the fixed effects for the presence of vascular MSU deposits on the MSU crystal volume in joint soft tissues was $0.4(p=0.35)$ in the feet and $1.21(p=0.03)$ in the knees. The presence of vascular DECT MSU deposits was associated with a 3.4-fold increase in MSU crystal volume in knee joint soft tissues throughout follow-up.

Conclusion: This study suggests that some vascular spots identified with DECT as MSU crystal deposition may be real and not artefacts. This correlation remains throughout follow-up in the knees. However, the comparable prevalence of vascular DECT MSU deposits between gout patients and controls, the systematic co-existence of vascular calcifications and the uneven regression under urate-lowering therapy requires further analysis to determine which DECT spots are artefacts and which are not.

References:

[1] Dual-Energy Computed Tomography Detection of Cardiovascular Monosodium Urate Deposits in Patients With Gout. Klauser AS, Halpern EJ, Strobl S, Gruber J, Feuchtner G, Bellmann-Weiler R, Weiss G, Stofferin H, Jaschke W.

Disclosure of Interests: Tristan Pascart Grant/research support from: Research Grant Horizon Pharma, Consultant of: Novartis, BMS, Sanofi, Pfizer,, Speak ers bureau: Novartis, BMS, Paul Carpentier: None declared, Laurène Norberciak: None declared, Julie Legrand: None declared, Eric Houvenagel Speakers bureau: Janssen, Novartis, Fabio Becce: None declared, Jean-François Budzik: None declared

DOI: 10.1136/annrheumdis-2020-eular.4522

\section{Diagnostics and imaging procedures}

\begin{tabular}{|l|l}
\hline OP0176 & CHRONOLOGICAL ORDER OF DECREASE OF \\
SYNOVITIS, OSTEITIS AND TENOSYNOVITIS \\
IN ARTHRITIS PATIENTS RECEIVING FIRST \\
DMARD-TREATMENT
\end{tabular}

X. Matthijssen ${ }^{1}$, E. Niemantsverdriet ${ }^{1}$, S. Le Cessie ${ }^{1}$, A. Van der Helm-van Mil ${ }^{1,2} .{ }^{1}$ Leiden University Medical Center (LUMC), Leiden, Netherlands; ${ }^{2}$ Erasmus MC, Rotterdam, Netherlands

Background: During the last decennium advanced imaging modalities have refined our understanding of the tissues involved in $\mathrm{RA}$ and have shown that not only joints but also bones and tendons can be inflamed at diagnosis. However, the time-order of decrease of these inflammatory features after initiation of DMARDs is unknown. Whether this differs for ACPA-positive and ACPA-negative patients is also unknown

Objectives: To achieve better understanding of the time order in which the different inflamed tissues (joint, tendon, bone) respond to DMARD-treatment and whether this differs between ACPA-subgroups.

Methods: 216 consecutive patients with early undifferentiated or rheumatoid arthritis, who received DMARD-treatment, were studied. Unilateral 1.5 Tesla contrast-enhanced MRIs of MCPs, wrists and MTPs were performed at baseline (before treatment) and after 4, 12 and 24 months. MRIs were scored for synovitis, osteitis and tenosynovitis in line with the RAMRIS, in known time-order but blinded for clinical data. Data of 4 serial time-points (three time intervals) were studied with autoregressive cross-lagged models. These models evaluated the influence of two time patterns in one model: 1) a simultaneous pattern ("extra change in one inflammatory feature was associated with extra change in another feature") and 2) a subsequent pattern ("change in one inflammatory feature preceded change in another feature"). All analyses were repeated stratified for ACPA-status (anti-CCP2)

Results: In all patients, all combinations of inflammatory features showed significant simultaneous decrease in all time intervals $(0-4 / 4-12 / 12-24$ months; all $\mathrm{p}<0.05)$. In addition to simultaneous changes there were also time orders identified: synovitis change between $0-4$ months preceded tenosynovitis change between $4-12$ months $(p=0.03)$ and synovitis change between 4 - 12 months preceded tenosynovitis change between months 12 - 24 months $(\mathrm{p}=0.02)$.

When considering ACPA-negative and ACPA-positive patients separately, similar results were obtained. In addition, in ACPA-positive patients, synovitis change between 4 - 12 months preceded osteitis change at $12-24$ months $(p=0.002)$; this was significantly different from ACPA-negative patients $(p<0.001)$.

Conclusion: This study increased the understanding of the response to treatment on tissue level. In addition to simultaneous decrease of synovitis, osteitis and tenosynovitis, also time orders of inflammation decrease were identified. 
These differed between ACPA-subgroups, implying a different interaction of synovium and bone in these patients.

Disclosure of Interests: None declared

DOI: 10.1136/annrheumdis-2020-eular.2157

\section{OP0177 PROPERTIES AND LOCATIONS OF COLOR-CODED DUAL ENERGY CT LESIONS IN GOUT PATIENTS - A SYSTEMATIC EVALUATION}

S. Nysom Christiansen ${ }^{1}$, F. C. Müller ${ }^{2}$, M. Ǿstergaard ${ }^{1}$, O. Slot ${ }^{1}$, J. Møllenbach Møller ${ }^{2}$, H. F. Børgesen ${ }^{2}$, K. K. Gosvig ${ }^{2}$, L. Terslev ${ }^{1} .{ }^{1}$ Rigshospitalet,

Copenhagen Center for Arthritis Research, Center for Rheumatology and Spine Diseases, Glostrup, Denmark; ${ }^{2} \mathrm{Herlev}$ Hospital, Department of Radiology, Herlev, Denmark

Background: Dual energy CT (DECT) has diagnostic potential in gout patients. DECT can automatically colour-code presumed urate deposits based on radiodensity (Hounsfield Units, HU) and DECT ratio (difference in attenuation between high and low $\mathrm{kV}$ series) of lesions. However, other materials may imitate properties of urate deposits, most importantly calcium-containing material, dense tendons and artefacts, which may lead to misinterpretations. The characteristics of DECT lesions in gout patients have not yet been systematically investigated. Objectives: To evaluate the properties and locations of colour-coded DECT lesions in gout patients.

Methods: DECT were performed in patients with suspected gout. Patients were separated into gout and non-gout patients based on joint fluid microscopy findings. DECT of the hands, knees and feet were performed using default gout settings and colour-coded lesions were registered. Only location-relevant lesions were analysed (e.g. nail bed artefacts excluded). Mean density (mean of $\mathrm{HU}$ at $80 \mathrm{kV}$ and Sn150kV), mean DECT ratio, size and location of each lesion was determined. Subgroup analysis was performed post-hoc evaluating potential differences in properties and locations of lesions. Lesions were separated into groups according to properties (Figure 1, grey box): 1) Size - to separate artefacts characterised by small volume (possible artefacts). 2) DECT ratios-to separate calcium-containing material characterised by high DECT ratio (possible calcium-containing material). 3) Density - to separate dense tendons characterised by low DECT ratio and low $\mathrm{HU}$ values (possible dense tendons). Lesion fulfilling all urate characteristics (large volume, low DECT ratio, high density) were labelled definite urate deposits. Finally, for non-gout patients, properties of non-gout urate-imitation lesions (properties as definite urate deposits) were analysed.

Results: In total, 3918 lesions (all lesions) were registered in gout patients $(n=23)$, with mean DECT ratio 1.06 (SD 0.13 ), median density $160.6 \mathrm{HU}$ and median size 6 voxels (Figure 1, blue box). Lesions were seen in all analysed joints, most frequently MTP1 joints (medial side), knee joints and midtarsal joints (Figure 2a). Tendon affections were also common, especially in the knee tendons (patella and quadriceps), malleolus-related tendons (e.g. peroneus and tibialis posterior) and the Achilles tendons (Figure 2a).

Subgroup analyses showed that definite urate deposits (figure $2 \mathrm{~b}$ ) were found at the same locations as all lesion in gout patients (figure 2a), with the four most common sites being MTP1 joints, midtarsal joints, and quadriceps and patella tendons (Figure 2b). Possible dense tendon lesions had a mean $\mathrm{HU}$ value of 156.5 $\mathrm{HU}-$ markedly higher than expected for dense tendons $(<100 \mathrm{HU})$-and lesion-locations were similar to definite urate deposits (data not shown), indicating that they primarily consisted of true urate deposits. In contrast, possible calcium-containing material and non-gout urate-imitating lesions had distinctly different properties (ratios 1.33 and 1.20, respectively) (Figure 1, yellow and orange box). Furthermore, the locations of these lesions were different from definite urate deposits since they were primarily found in a few weight-bearing joints (knee, midtarsal and talocrural including malleolus regions) and tendons (Achilles and quadriceps), whereas no lesions were found in either MTP1 joints or patella tendons (figure 2c).

Conclusion: DECT color-coded lesions in gout patients are heterogeneous in properties and locations. Subgroup analyses found that locations such as MTP1 joints and patella tendons were characterised by almost only showing definite urate deposits. A sole focus on these regions in the evaluation of gout patients may therefore improve specificity of DECT scans.

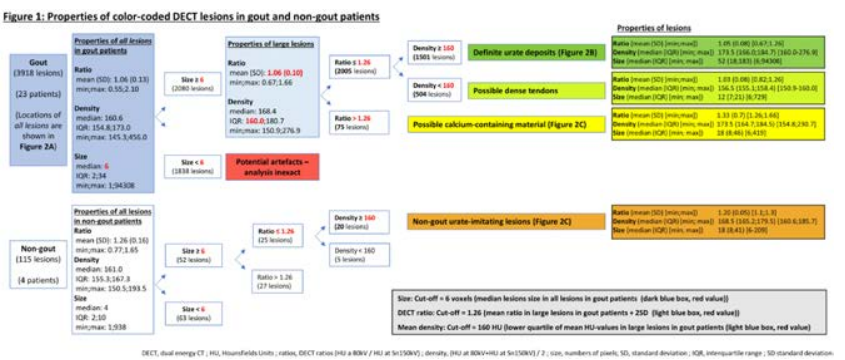

Figure 2

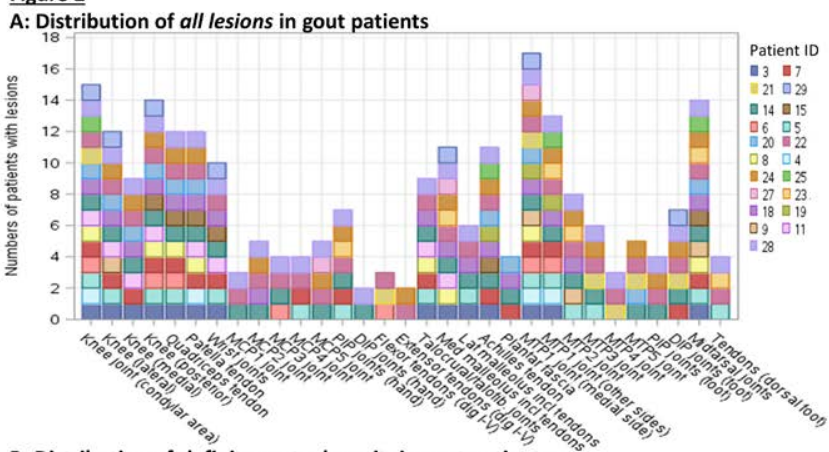

B: Distribution of definite urate deposits in gout patients

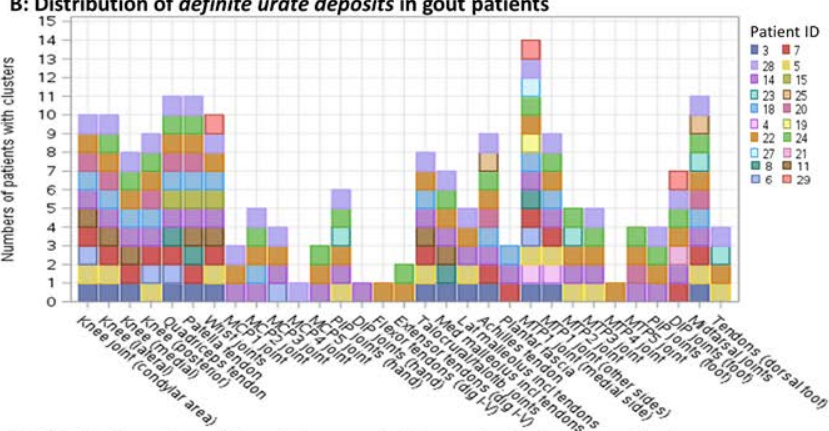

C: Distribution of possible calcium-containing material in gout patients and non-gout urate-imitating lesions

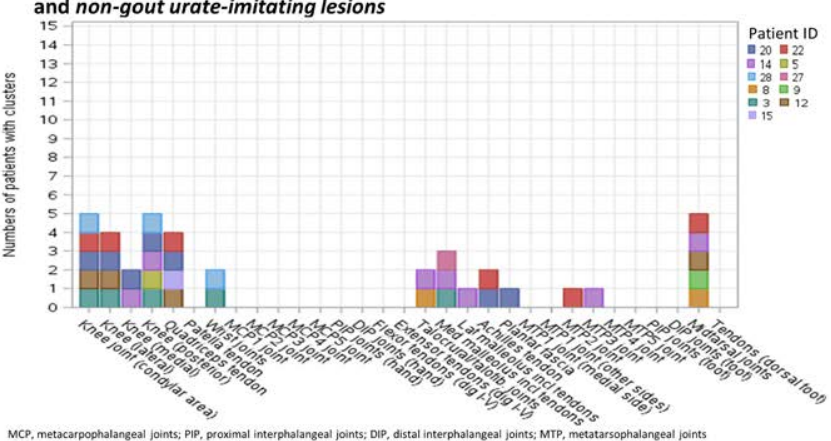

Disclosure of Interests: Sara Nysom Christiansen Speakers bureau: SNC has received speaker fees from Bristol Myers Squibb (BMS) and General Electric (GE)., Felix C Müller Employee of: Siemens Healthineers., Mikkel Ǿstergaard Grant/research support from: AbbVie, Bristol-Myers Squibb, Celgene, Merck, and Novartis, Consultant of: AbbVie, Bristol-Myers Squibb, Boehringer Ingelheim, Celgene, Eli Lilly, Hospira, Janssen, Merck, Novartis, Novo Nordisk, Orion, Pfizer, Regeneron, Roche, Sandoz, Sanofi, and UCB, Speakers bureau: AbbVie, Bristol-Myers Squibb, Boehringer Ingelheim, Celgene, Eli Lilly, Hospira, Janssen, Merck, Novartis, Novo Nordisk, Orion, Pfizer, Regeneron, Roche, Sandoz, Sanofi, and UCB, Ole Slot: None declared, Jakob Møllenbach Møller: None declared, Henrik F Børgesen: None declared, Kasper K Gosvig: None declared Lene Terslev Speakers bureau: LT declares speakers fees from Roche, MSD BMS, Pfizer, AbbVie, Novartis, and Janssen. DOI: 10.1136/annrheumdis-2020-eular.1757

\begin{tabular}{l|l}
\hline OP0178 & IMAGING NEOANGIOGENESIS IN RHEUMATOID \\
ARTHRITIS (INIRA): WHOLE-BODY SYNOVIAL UPTAKE \\
OF A 99MTC-LABELLED RGD PEPTIDE IS HIGHLY \\
CORRELATED WITH POWER DOPPLER ULTRASOUND
\end{tabular}

L. Attipoe $^{1}$, S. Subesinghe ${ }^{2}$, C. Blanco-Gil' ${ }^{2}$, M. Opena ${ }^{2}$, M. Rosser ${ }^{3}$, G. Cook $^{1}$, A. Cope ${ }^{1}$, T. Garrood ${ }^{2}{ }^{1}$ King's College London, London, United Kingdom; ${ }^{2}$ Guy's and St Thomas' NHS Foundation Trust, London, United Kingdom; ${ }^{3}$ Serac Life Sciences, London, United Kingdom

Background: Power Doppler ultrasound (PDUS) is superior to clinical examination in detecting synovitis in patients with rheumatoid arthritis (RA). Although dynamic and cheap it is impractical to scan large numbers of joints in routine clinical settings. MRI, whilst sensitive for synovitis, is expensive and routine use is limited to targeted joints. Bone scintigraphy produces whole body images but due to limited specificity is not routinely used. 